\title{
FRACTAIS: UMA APLICAÇÃO DO TEOREMA DE NAPOLEÃO
}

\section{FRACTALS: AN APPLICATION OF NAPOLEON'S THEOREM}

José Augusto da Costa Jacomeli, Fernando Pereira de Souza e Eugenia Brunilda Opazo.

Universidade Federal de Mato Grosso do Sul - UFMS. E-mail: gu_jacomeli@outlook.com

RESUMO - A geometria fractal permite a interdisciplinaridade com diversos temas da Matemática e de outras áreas, desde as ciências naturais às econômicas e à tecnologia. $\mathrm{O}$ presente trabalho aborda a construção de um fractal como aplicação de um teorema muito importante da geometria plana, conhecido como Teorema de Napoleão. Aplicamos o Teorema de Napoleão em um triângulo equilátero obtendo a famosa estrela de Davi, as pontas da estrela formam novos triângulos equiláteros e o Teorema é novamente utilizado, este processo é aplicado sucessivamente e o resultado obtido é um fractal que se assemelha com a curva de Koch. O objetivo deste trabalho é encontrar o perímetro e a área do fractal, para isto, conceitos de geometria plana, semelhança e congruência de triângulos e também alguns tópicos de matemática discreta, sequências e séries numéricas serão necessários para atingir tal objetivo.

Palavras-chave: geometria; fractais, Teorema de Napoleão

ABSTRACT - Fractal geometry allows for interdisciplinary with various topics in mathematics and other fields, from the natural sciences to economics and technology. The present work approaches the construction of a fractal as an application of a very important theorem of plane geometry, known as Napoleon's Theorem. We apply Napoleon's theorem in an equilateral triangle obtaining the famous Star of David, the star's ends form new equilateral triangles and the theorem is used again, this process is applied successively and the result obtained is a fractal that resembles the curve of Koch. The objective of this work is to find the perimeter and the fractal area, for this, concepts of plane geometry, similarity and congruence of triangles and also some discrete math topics, sequences and numerical series will be necessary to achieve this goal.

Keywords: geometry; fractals, Napoleon's theorem 


\section{INTRODUÇÃO}

O Teorema de Napoleão é um dos clássicos teoremas na área de geometria que apresenta diversas provas, generalizações, variantes e que por muitas vezes vem sido esquecido e pouco estudado em cursos de graduação em Matemática.

Napoleão Bonaparte (1769 - 1821) famoso pelo seu trabalho na área militar e na área política apresentava um grande interesse na área das ciências, principalmente na Matemática. Apesar de não haver provas que comprove que Napoleão é o verdadeiro autor, o Teorema de Napoleão tem se esse nome em homenagem ao soldado.

A Geometria Fractal, uma área matemática pouca estudada durante os cursos de graduação, tem o objetivo de estudar os fractais. Os fractais são figuras na qual cada uma apresenta uma característica única durante todo seu domínio, de modo que cada parte da figura é encontrada uma imagem de si própria. Um dos exemplos mais conhecidos de fractais é a Curva de Koch (Floco de Neve), como pode ser visto na Figura 1.

Figura 1. Floco de Neve.
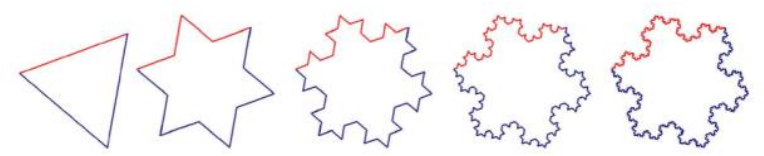

Fonte: Nunes 2006

Neste Trabalho, utilizaremos o Teorema de Napoleão na construção de um fractal, aplicaremos o Teorema de Napoleão em um triângulo equilátero e depois aplicaremos novamente nos triângulos menores formados nas extremidades da figura e assim sucessivamente. A figura encontrada se assemelha com a famosa Curva de Koch, porém, o fractal formado não foi encontrado nas bibliografias atuais.

Desse modo, o objetivo do trabalho é verificar se é possível aplicarmos o teorema infinitas vezes em um triângulo equilátero, e se possível encontrarmos a área e o perímetro do fractal formado.

\section{METODOLOGIA}

A O presente trabalho está incluso em uma atividade de pesquisa individual do grupo PET Conexões de Saberes Matemática UFMS/CPTL, que tem o objetivo de relembrar e/ou aprofundar o conhecimento em conceitos matemáticos que por muitas vezes são esquecidos ou poucos estudados durante a graduação.

O trabalho surgiu após uma pesquisa sobre Teoremas matemáticos esquecidos, na qual foi estudado o conceito $e$ a demonstração do Teorema de Napoleão. Após isso, aplicamos o teorema em um triângulo equilátero de lado $l$, assim obtemos a seguinte figura:

Figura 2. Estrela de Davi.

Fonte: O Autor.

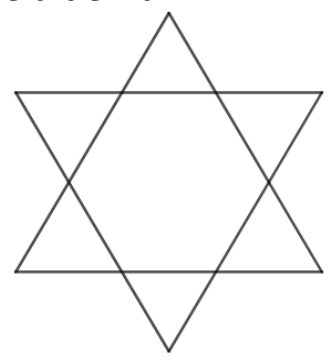

Dessa forma, a figura 2 é conhecida como sendo a famosa Estrela de Davi símbolo do judaísmo. Que consiste em dois triângulos iguais com posições opostas, assim pode ser obtido a área e o perímetro da estrela em função do lado $l$.

Os triângulos menores presentes nas pontas da estrela são triângulos equiláteros, possibilitando a aplicação do teorema novamente nesses triângulos, e assim sucessivamente, formando assim um fractal. Assim formamos as seguintes figuras conforme cada estágio/aplicação do Teorema: 
Figura 3. Estágio 1.

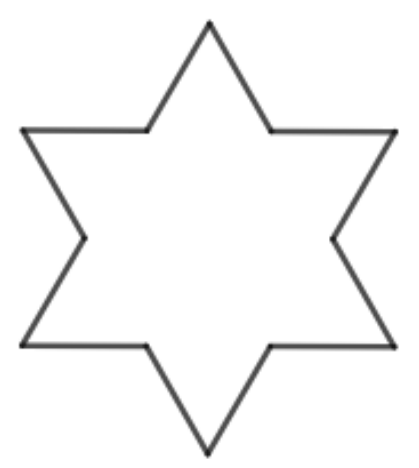

Fonte: O Autor.

Figura 4. Estágio 2.

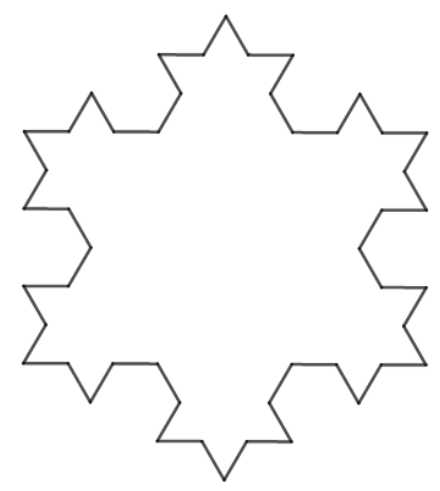

Fonte: O Autor.

Figura 5. Estágio 3.

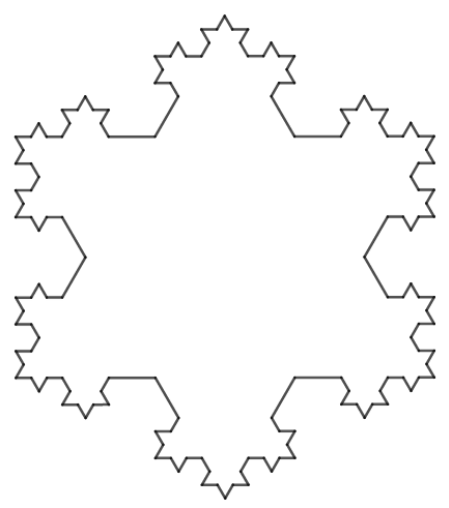

Fonte: O Autor.

\section{RESULTADOS E DISCUSSÕES}

\subsection{TEOREMA DE NAPOLEÃO}

A demonstração do Teorema pode ser feita de diversas maneiras, mas aqui provamos por propriedades de semelhança de triângulos.

Teorema de Napoleão: Seja $\triangle A B C$ um triângulo arbitrário, se em seus lados forem construídos triângulos equiláteros, os ortocentros desses triângulos formará um novo triângulo equilátero. A ilustração do teorema pode ser visto na imagem a seguir:
Figura 6. Teorema de Napoleão.

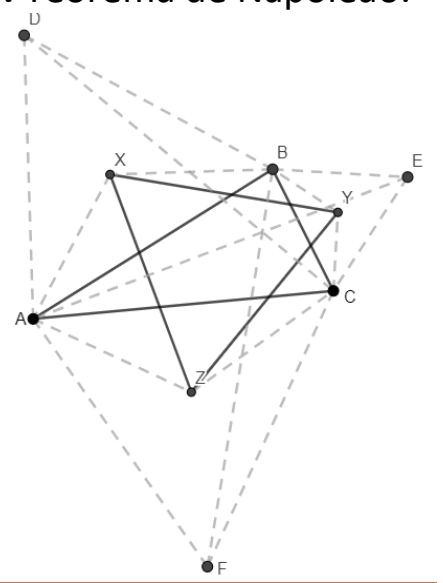

Fonte: O Autor.

Dem: Seja $\triangle A B C$ o triângulo original. Escolha $D, E$ e $F$ pontos exteriores ao triângulo $\triangle A B C$ tais que $\triangle A D B_{y} \triangle B E C$ e $\triangle A F C$ sejam triângulos equiláteros, com centroides $X, Y$ e $Z$, respectivamente.

Do fato dos triângulos $\triangle F B C$ e $\triangle A E C$ serem equiláteros, temos:

$$
\overline{F C}=\overline{A C} \text { e } \overline{E C}=\overline{B C} \text {, }
$$

e

$$
\mathrm{F} \hat{C} B=60^{\circ}+A \hat{C} B=A \hat{C} E \text {. }
$$

Desta forma, os triângulos $\triangle A E C$ e $\triangle F B C$ são congruentes, e assim obtemos $\overline{A E} \equiv \overline{F B}, \quad$ analogamente usando os triângulos $\triangle D B C$ e $\triangle A E C$ mostramos que $\overline{A E} \equiv \overline{D C}$.

Notemos que como $X, Y$ e $Z$ são centroides dos triângulos equiláteros $\triangle A D B$, $\triangle B E C$ e $\triangle A F C_{\text {, }}$ então:

$$
|\overline{Z C}|=\frac{\sqrt{3}}{3}, \quad|\overline{C Y}|=\frac{\sqrt{3}}{3}|\overline{B C}| .
$$

Dessa forma, temos que:

$$
\frac{|\overline{Z C}|}{|\overline{F C}|}=\frac{|\overline{C Y}|}{|\overline{B C}|}=\frac{\sqrt{3}}{3}
$$

e ainda, como

$$
\begin{aligned}
& Z \hat{C} Y=Z \hat{C} A+A \hat{C} B+B \hat{C} Y=30^{\circ}+A \hat{C} B+ \\
& 30^{\circ}=F \hat{C} B
\end{aligned}
$$

obtemos que os triângulos $\triangle F C B$ e $\triangle Z Y C$ são semelhantes, o que nos dá

$$
\frac{|\overline{Z C}|}{|\overline{F C}|}=\frac{|\overline{C Y}|}{|\overline{B C}|}=\frac{|\overline{Y Z}|}{|\overline{B F}|}=\frac{\sqrt{3}}{3} \text {. }
$$

Agora, temos que:

$$
A \widehat{B} E=A \widehat{B} Y+Y \widehat{B} E=A \widehat{B} Y+30^{\circ} \equiv X \widehat{B} Y,
$$

e 
$|\overline{B Y}|=\frac{\sqrt{3}}{3}|\overline{B E}|, \quad|\overline{B X}|=\frac{\sqrt{3}}{3}|\overline{B A}|$.

Isto nos dá que os triângulos $\triangle A B E$ e

$\triangle X B Y$ são semelhantes, então temos que:

$$
\frac{|\overline{A E}|}{|\overline{X Y}|}=\frac{|\overline{B A}|}{|\overline{B X}|}=\frac{|\overline{B E}|}{|\overline{B Y}|}
$$

Por último temos:

$D \hat{A C}=D \hat{A} X+X \hat{A} C=30^{\circ}+X \hat{A} C=X \hat{A} Z$, e

$$
|\overline{A Z}|=\frac{\sqrt{3}}{3}|\overline{A C}|, \quad|\overline{X A}|=\frac{\sqrt{3}}{3}|\overline{D A}| .
$$

Logo, os triângulos $\triangle D A C$ e $\triangle X A Z$ são semelhantes, o que nos dá:

$$
\frac{|\overline{D C}|}{|\overline{X Z}|}=\frac{|\overline{D A}|}{|\overline{A X}|}=\frac{|\overline{A C}|}{|\overline{A Z}|}
$$

Agora, de (I), (II) e (III), temos:

$$
\frac{|\overline{D C}|}{|\overline{X Z}|}=\frac{|\overline{B F}|}{|\overline{Y Z}|}=\frac{|\overline{A E}|}{|\overline{X Y}|}
$$

Mas, como $|\overline{B F}|=|\overline{D C}|=|\overline{A E}|$ então teremos que $|\overline{X Z}|=|\overline{Y Z}|=|\overline{X Y}|$. Portanto, o triângulo $\triangle X Y Z$ é equilátero.

\subsection{TEOREMA DE NAPOLEÃO NO TRIÂNGULO EQUILÁTERO}

Agora, supondo que o triângulo original $\triangle A B C$ seja equilátero, o Teorema de Napoleão nos garante que o triângulo formado $\triangle X Y Z$ também será equilátero. Agora, utilizando também propriedades de semelhança de triângulos temos que os triângulos $\triangle A B C$ e $\triangle X Y Z$ serão congruentes.

Figura 7. Demonstração do Teorema de Napoleão.

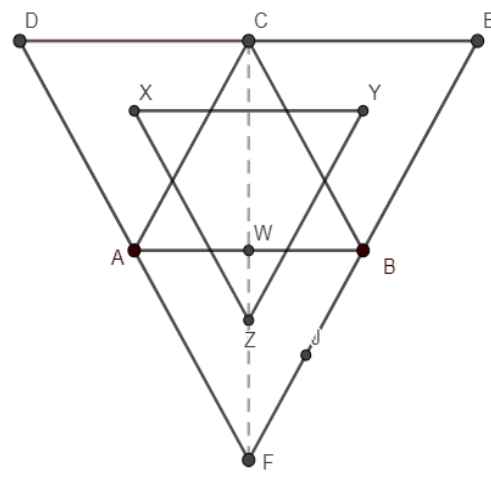

Fonte: O Autor.

Dem: Seja $\triangle A B C$ um triângulo equilátero, de $(I)$ da demonstração do teorema anterior, temos:

$$
\begin{aligned}
\frac{|\overline{Y Z}|}{|\overline{C F}|} & =\frac{|\overline{B Y}|}{|\overline{B C}|} \\
|\overline{Y Z}||\overline{B C}| & =|\overline{B Y}||\overline{C F}|,
\end{aligned}
$$

mas, $|\overline{B Y}|=\frac{\sqrt{3}}{3}|\overline{B C}|$, então:

$$
\begin{gathered}
|\overline{Y Z}||\overline{B C}|=\frac{\sqrt{3}}{3}|\overline{B C}||\overline{C F}| \\
|\overline{Y Z}||\overline{B C}|-\frac{\sqrt{3}}{3}|\overline{B C}||\overline{C F}|=0 \\
|\overline{B C}|\left(|\overline{Y Z}|-\frac{\sqrt{3}}{3}|\overline{C F}|=0\right. \\
|\overline{Y Z}|=\frac{\sqrt{3}}{3}|\overline{C F}| \\
|\overline{C F}|=\frac{3 \sqrt{3}}{3}|\overline{Y Z}| .
\end{gathered}
$$

Sendo $W$ ponto médio de $|\overline{A C}|$, então $|\overline{B W}|$ e $|\overline{F W}|$ são a altura relativa dos triângulos $\triangle A B C$ e $\triangle A C F$ respectivamente, e como o $\triangle A B C$ é equilátero, temos que:

$$
|\overline{B W}|=\frac{1}{2}|\overline{A C}|=\frac{1}{2}|\overline{B C}|
$$

Logo, utilizando o Teorema de Pitágoras, temos:

$$
\begin{gathered}
|\overline{B C}|^{2}=|\overline{C W}|^{2}+|\overline{B W}|^{2} \\
|\overline{B C}|^{2}=|\overline{C W}|^{2}+\frac{1}{4}|\overline{B C}|^{2} \\
|\overline{B C}|^{2}\left(1-\frac{1}{4}\right)=|\overline{C W}|^{2} \\
\frac{3}{4}|\overline{B C}|^{2}=|\overline{C W}|^{2} \\
\frac{\sqrt{3}}{2}|\overline{B C}|=|\overline{C W}|,(I)
\end{gathered}
$$

e ainda, sendo $W$ também ponto médio de $|\overline{B F}|$, então:

$$
\begin{gathered}
|\overline{C W}|=\frac{1}{2}|\overline{C F}|=\frac{1}{2} \times \frac{3 \sqrt{3}}{3}|\overline{Y Z}|=\frac{\sqrt{3}}{2}|\overline{Y Z}|,(I I) \\
\text { Assim, de }(I) \mathrm{e}(I I), \text { temos } \\
\frac{\sqrt{3}}{2}|\overline{Y Z}|=\frac{\sqrt{3}}{2}|\overline{B C}| \\
|\overline{Y Z}|=|\overline{B C}| .
\end{gathered}
$$

Portanto,

$$
\triangle X Y Z \equiv \triangle A B C .
$$

Assim, a primeira aplicação do Teorema de Napoleão em um triângulo equilátero nos dá a seguinte figura: 
Figura 8. Estrela de Davi.

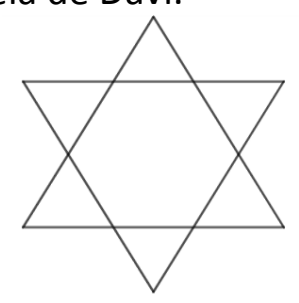

Fonte: O Autor

A figura consiste em 2 triângulos congruentes de posições opostas, o que corresponde a famosa Estrela de Davi símbolo do judaísmo.

\subsection{DECOMPOSIÇÃO DA FIGURA}

Assim, observando a figura 7, vemos que ela consiste em 6 triângulos menores e 1 hexágono central. Vamos provar que os triângulos são equiláteros e o hexágono central é regular.

Figura 9. Demonstração Triângulos Menores.

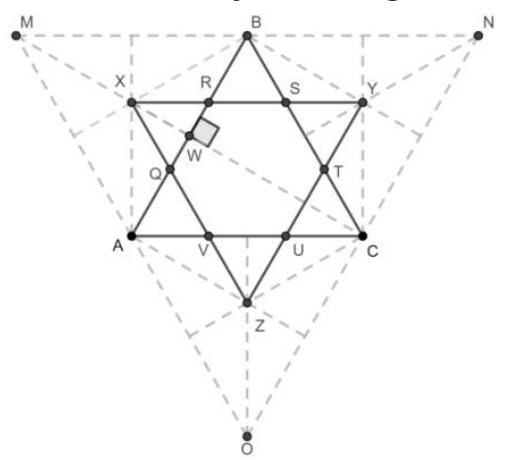

Fonte: O Autor.

Seja $W$ o ponto médio do segmento $\overline{A B}$, assim, $\overline{C W}$ será a altura relativa do triângulo $\triangle A B C$, e sabendo que $\overline{X W}$ é colinear a $\overline{X C}$, pois temos que $X$ é o ortocentro do triângulo equilátero gerado pelo lado $\overline{A B}, \quad$ como $\widehat{C W} \bar{B}=90^{\circ}$, consequentemente $\widehat{X W} \bar{R}=90^{\circ}$. Dessa mesma forma, a bissetriz do $\triangle X Y Z$ relativa ao ângulo $\widehat{Z X Y}$ também será colinear ao segmento $\overline{X W}$, assim teremos que o ângulo $\widehat{W X} \bar{R}=30^{\circ}$, assim sendo o ângulo $\widehat{X R W}=60^{\circ}$, assim:

$$
\widehat{Q X \bar{R}}=\widehat{\bar{X} R \bar{Q}}=\widehat{R Q \bar{X}}=60^{\circ} \text {. }
$$

Portanto, o triângulo menor $\triangle Q X R$ é equilátero. Analogamente, teremos que os 6 triângulos menores serão equiláteros.
Como vimos, os ângulos $\widehat{W X} \bar{R}=\widehat{C X S}=30^{\circ}$ e ainda, da mesma forma, obteremos que $\mathrm{o}$ ângulo $\widehat{W C T}=\widehat{X C S}=30^{\circ}$, então

$$
\begin{gathered}
\overrightarrow{C X S}+\overrightarrow{X C S}+\overrightarrow{C S X}=180^{\circ} \\
\overrightarrow{C S X}=120^{\circ} .
\end{gathered}
$$

Portanto, semelhantemente teremos que todos os ângulos internos do hexágono central serão de $120^{\circ}$, ou seja, o hexágono central da estrela será um hexágono regular. Dessa forma, temos que os triângulos equiláteros menores possuíram o mesmo tamanho.

\subsection{PERÍMETRO E ÁREA DA ESTRELA}

Seja um triângulo $\triangle A B C$ equilátero de lado $l$, logo como vimos, ao aplicarmos o teorema, nos resultará na Estrela de Davi, que se decompõe em 6 triângulos menores equiláteros iguais e 1 hexágono regular central.

Seja o lado $\overline{A B}$ do triângulo, logo o lado é dividido em 3 triângulos menores, como os triângulos possuem o mesmo tamanho, então o lado de cada triângulo menor será $\frac{l}{3}$. Então, o perímetro da estrela será:

$$
P=12 \cdot \frac{l}{3}=4 l
$$

Como vimos, a estrela é formada por 2 triângulos iguais com posições opostas, então a área da estrela é formada pela a área dos dois triângulos maiores menos a área hexágono central. Logo, como o lado do hexágono mede $\frac{l}{3} \mathrm{e} \frac{\sqrt{3} l}{2}$ a altura dos triângulos maiores. Então:

$$
A=2 \frac{\frac{\sqrt{3} l^{2}}{2}}{2}-\frac{3\left(\frac{l}{3}\right)^{2} \sqrt{3}}{2}=\frac{\sqrt{3} l^{2}}{3} .
$$

\subsection{APLICAÇÃO INFINITA DO TEOREMA DE NAPOLEÃO}

Como vimos, ao aplicarmos a $1^{\circ}$ vez o Teorema em um triângulo equilátero, surge 6 triângulos menores equiláteros iguais. Dessa forma, podemos aplicar novamente o Teorema nesses triângulos, e assim sucessivamente aplicando nos triângulos formados no exterior da figura, formando um 
fractal. Assim, sabendo que em cada estágio é uma nova aplicação do Teorema, e focando apenas em uma parte da figura e depois generalizando, temos:

Figura 10. $1^{\circ}$ Aplicação do Teorema.

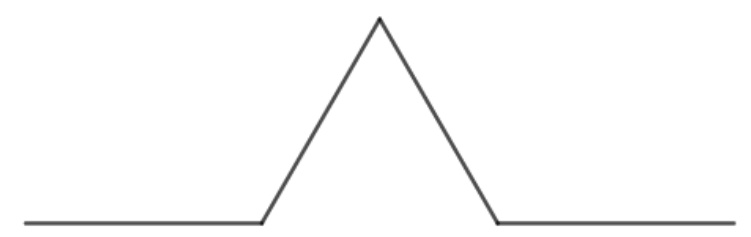

Fonte: O Autor.

Figura 11. $2^{\circ}$ Aplicação do Teorema.

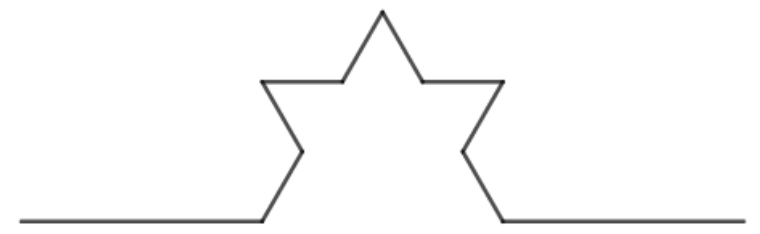

Fonte: O Autor

Figura $12.3^{\circ}$ Aplicação do Teorema.

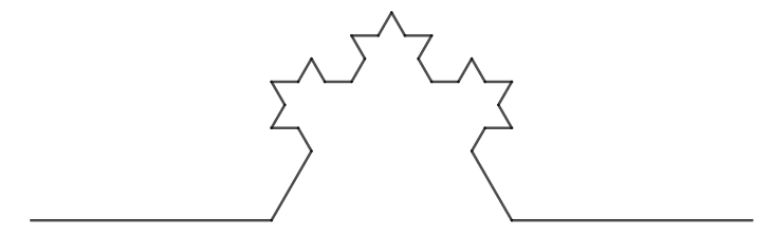

Fonte: O Autor

Figura 13. $4^{\circ}$ Aplicação do Teorema.

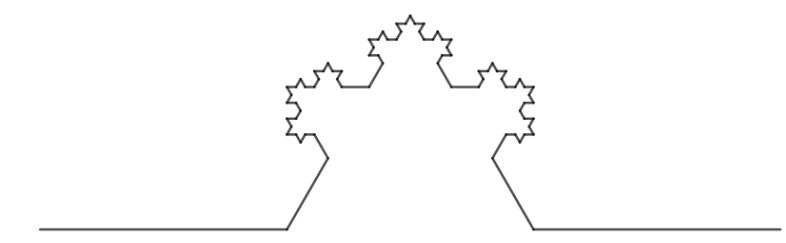

Fonte: O Autor.
Figura 14. $5^{\circ}$ Aplicação do Teorema.

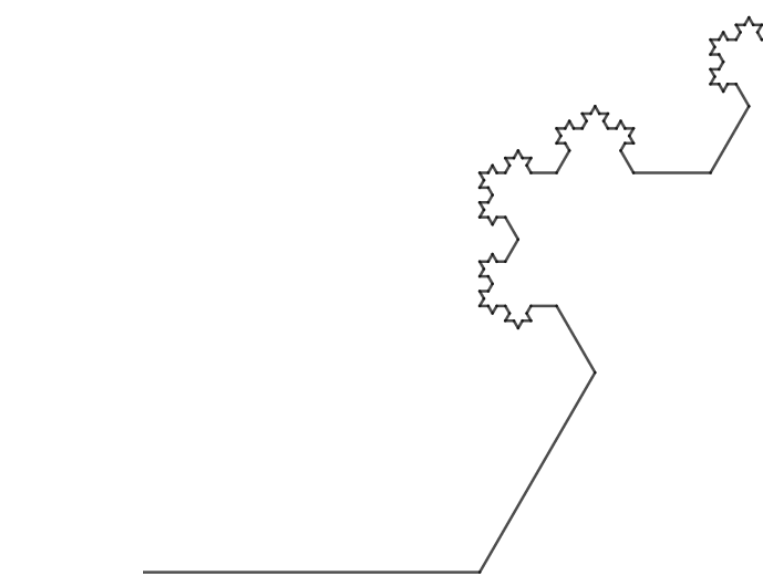

Fonte: O Autor.

O triângulo $\triangle A B C$ inicial possui lado $l$, então cada estágio os lados novos irão ter proporção de $1 / 3$ em relação ao lado do estágio anterior, e ainda a cada estágio irão ser fixados alguns lados que não se dividem, assim construímos a seguinte tabela:

Tabela 1. Proporção dos lados da figura.

\begin{tabular}{|c|c|c|c|c|}
\hline Estágio & $\begin{array}{c}\text { Lados } \\
\text { Fixos }\end{array}$ & Proporção & $\begin{array}{c}\text { Lados } \\
\text { Novos }\end{array}$ & Proporção \\
\hline 1 & - & - & $2^{2} \cdot 3$ & $1 / 3$ \\
\hline 2 & - & - & $2^{4} \cdot 3$ & $\frac{1}{3^{2}}$ \\
\hline 3 & $2^{2} 3$ & $\frac{1}{3^{2}}$ & $2^{4} 3^{2}$ & $\frac{1}{3^{3}}$ \\
\hline 4 & $2^{2} 3$ & $\frac{1}{3^{2}}$ & $2^{4} 3^{2}$ & $\frac{1}{3^{4}}$ \\
\hline 5 & $2^{2} 3^{2}$ & $\frac{1}{3^{a}}$ & & \\
& $2^{2} 3$ & $\frac{1}{3^{2}}$ & $2^{4} 3^{4}$ & $\frac{1}{3^{5}}$ \\
& $2^{2} 3^{2}$ & $\frac{1}{3^{a}}$ & & \\
\hline
\end{tabular}

Fonte: O Autor.

Dessa forma, seja $P_{n}$ o perímetro para o $n^{\circ}$ estágio, então:

$$
\begin{aligned}
& P_{1}=2^{2} 3 \frac{l}{3} ; \\
& P_{2}=2^{4} 3 \frac{l}{3^{2}} \\
& P_{3}=2^{2} 3 \frac{l}{3^{2}}+2^{4} 3^{2} \frac{l}{3^{3}}
\end{aligned}
$$




$$
\begin{aligned}
P_{4} & =2^{2} 3 \frac{l}{3^{2}}+2^{2} 3^{2} \frac{l}{3^{3}}+2^{4} 3^{3} \frac{l}{3^{4}} \\
P_{5} & =2^{2} 3 \frac{l}{3^{2}}+2^{2} 3^{2} \frac{l}{3^{3}}+2^{2} 3^{3} \frac{l}{3^{4}}+2^{4} 3^{4} \frac{l}{3^{5}} \\
P_{n} & =\sum_{k=1}^{n-2} 2^{2} 3^{k} \frac{l}{3^{k+1}}+2^{4} 3^{n-1} \frac{l}{3^{n}}=\sum_{k=1}^{n-2} \frac{2^{2}}{3} l+\frac{2^{4} l}{3} \\
& =\frac{2^{2} l}{3}\left(n-2+2^{2}\right)=\frac{4 l}{3}(n+2),
\end{aligned}
$$

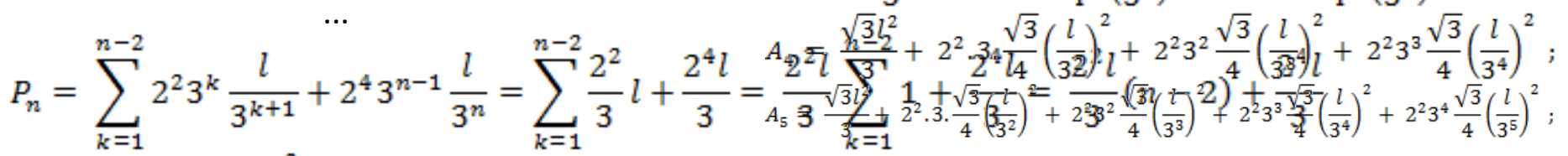

Logo, o perímetro da fractal, será

$$
P=\lim _{n \rightarrow \infty} P_{n}=\lim _{n \rightarrow \infty} \frac{4 l}{3}(n+2)=+\infty \text {. }
$$

Dessa forma, temos que o perímetro do fractal diverge.

Agora, para encontrar a área da figura, temos que observar que sempre irá surgindo novos triângulos menores, então podemos ver a relação dos lados dos novos triângulos vendo a próxima figura que contém a tabela:

Tabela 2. Proporção dos Triângulos da figura.

\begin{tabular}{|c|c|c|c|c|}
\hline Estágio & $\begin{array}{l}\text { Triângulos } \\
\text { Fixos }\end{array}$ & $\begin{array}{l}\text { Propor- } \\
\text { ção }\end{array}$ & $\begin{array}{l}\text { Triângulos } \\
\text { Novos }\end{array}$ & $\begin{array}{l}\text { Propor- } \\
\text { ção }\end{array}$ \\
\hline 1 & - & - & 2.3 & $\frac{1}{3}$ \\
\hline 2 & 2.3 & $\frac{1}{3}$ & $2^{2} \cdot 3$ & $\frac{1}{3^{2}}$ \\
\hline 3 & 2.3 & $\frac{1}{3}$ & $2^{2} 3^{2}$ & $\frac{1}{3^{a}}$ \\
\hline 4 & 2.3 & $\frac{1}{3}$ & $2^{2} 3^{2}$ & $\frac{1}{3^{4}}$ \\
& $2^{2} \cdot 3$ & $\frac{1}{3^{2}}$ & & \\
\hline 5 & 2.3 & $\frac{1}{3}$ & $2^{2} 3^{4}$ & $\frac{1}{3^{5}}$ \\
& $2^{2} \cdot 3$ & $\frac{1}{3^{2}}$ & & \\
& $2^{2} 3^{2}$ & $\frac{1}{3^{a}}$ & & \\
& $2^{2} 3^{a}$ & $\frac{1}{3^{4}}$ & & \\
\hline
\end{tabular}

Fonte: O Autor.

Mas, temos a área de um triângulo equilátero é de $\frac{\sqrt{3}}{4} l^{2}$, então sabendo que o estágio 1 é a Estrela de Davi, uma área já conhecida, temos:

$A_{1}=\frac{\sqrt{3} l^{2}}{3}$

$$
A_{2}=\frac{\sqrt{3} l^{2}}{3}+2^{2} \cdot 3 \cdot \frac{\sqrt{3}}{4}\left(\frac{l}{3^{2}}\right)^{2} \text {; }
$$$$
A_{3}=\frac{\sqrt{3} l^{2}}{3}+2^{2} \cdot 3 \cdot \frac{\sqrt{3}}{4}\left(\frac{l}{3^{2}}\right)^{2}+2^{2} 3^{2} \frac{\sqrt{3}}{4}\left(\frac{l}{3^{3}}\right)^{2} \text {; }
$$

$A_{n} \geq \frac{\sqrt[3]{3} l^{2}}{3}+\sqrt{3} l^{2}\left(3 \frac{l}{3^{4}}+3^{2} \frac{l}{3^{6}}+3^{3} \frac{l}{3^{8}}+3^{4} \frac{l}{3^{10}}+\cdots\right)=$

$$
\begin{gathered}
\frac{\sqrt{3} l^{2}}{3}+\sqrt{3} l^{2}\left(\frac{l}{3^{3}}+\frac{l}{3^{4}}+\frac{l}{3^{5}}+\frac{l}{3^{6}}+\cdots\right)= \\
\frac{\sqrt{3} l^{2}}{3}+\sqrt{3} l^{2} \sum_{k=1}^{n}\left(\frac{1}{3}\right)^{k+2}, \quad n \geq 2 .
\end{gathered}
$$

Mas, temos que $\sum_{k=1}^{n}\left(\frac{1}{3}\right)^{k+2}$, quando $\mathrm{n}$ tende ao infinito, é uma série geométrica com razão a, $-1<a<1$, então a série converge. Portanto, a área do fractal é dada por:

$A=\lim _{n \rightarrow \infty} A_{n}$

$$
\begin{aligned}
& =\lim _{n \rightarrow \infty}\left(\frac{\sqrt{3}}{3} l^{2}+\sqrt{3} l^{2} \sum_{k=1}^{n}\left(\frac{1}{3}\right)^{k+2}\right) \\
& =\frac{\sqrt{3}}{3} l^{2}+\frac{\sqrt{3}}{18} l^{2}=\frac{7 \sqrt{3}}{18} l^{2} .
\end{aligned}
$$

\section{CONCLUSÃO}

Ao aplicarmos o Teorema de Napoleão em um triângulo equilátero infinito vezes nos resultará em um fractal que é uma figura que ainda não tinha sido explorada. Então, no trabalho conseguimos explorar mais esse fractal mostrando que é possível fazer sua construção e descobrirmos que a figura formada pela superfície apresentará um perímetro infinito e uma área finita.

Portanto, no trabalho podemos explorar e relembrar propriedades e conceitos da geometria, da álgebra e do cálculo, que já foram esquecidas ou que não foram estudadas durante $o$ curso de graduação. Sendo assim, tivemos um desenvolvimento esperado para o objetivo da atividade do programa e, além disso, contribuindo para um melhor desenvolvimento nas matérias do curso de Matemática e para uma futura pósgraduação. 
5. REFERÊNCIAS

ALVES, D. S. Os Teoremas Esquecidos pelos

Professores de Geometria Plana do Ensino

Médio. 2015. Dissertação (Mestrado) Universidade Federal de Mato Grosso do Sul, Campo Grande, 2015.

BRITO, A. J.; CARVALHO, D. L. História da matemática em atividades didáticas: Utilizando a história no Ensino de Geometria. Natal: EDUFRN, 2005.

GONZAGA. G. C. S. Teorema de Napoleão: Origem, Demonstração e Aplicações. 2015. Dissertação (Mestrado) - Universidade Federal de Goiás, Goiânia, 2015.

MACEDO, D. M. R. Resgatando alguns Teoremas Clássicos da Geometria Plana. 2014. Dissertação (Mestrado)- Universidade Federal do Ceará, Juazeiro do Norte, 2014.

NUNES, R. S. R. Geometria Fractal e Aplicações. 2006. Dissertação (Mestrado) Faculdade de Ciências da Universidade do Porto, Departamento de Matemática Pura, Porto, 2006. 